\title{
DIABETES INSIPIDUS DUE TO HYPOPHYSITIS
}

\section{Agathi Vasileiou, Dimitra Pappa, Georgios Filippakopoulos, loannis Gkountios, Alexandra Bargiota}

Endocrinology, Diabetes and Metabolic Diseases Department, University Hospital of Larisa, Hellas

Introduction: Hypophysitis is a rare entity, more common in women in pregnancy and the postpartum period and can cause various symptoms such as headache, hypopituitarism and visual disturbances. Stalk thickening and homogeneous enhancement of the gland are the characteristic magnetic resonance imaging (MRI) findings. The diagnosis is based on the clinical picture and on follow up MRIs. Usually involves the anterior pituitary alone, whereas isolated-posterior-pituitary-involvement is quite rare.

We present here two cases of diabetes insipidus due to hypophysistis.

Case reports: A 35-year-old woman presented with a two month history of headache and the last fortnight she developed polydipsia, polyuria and nocturia. Her past medical history, except an autoimmune thyroiditis, was unremarkable. Clinical and biochemical findings were in accordance with diabetes insipidus. The anterior pituitary function was intact. Pituitary MRI showed enlargement of posterior lobe, stalk thickening and homogeneous enhancement of pituitary's signal after gadolinium administration (Fig1). She was treated with prednisolone 40 mgrs tapered over 3 months. The symptoms gradually subsided, with a significant improvement of her imaging findings at 3 months (Fig2).

A 46-year-old woman, with history of hyperlipidemia, developed suddenly symptoms of extreme polyuria and polydipsia and presented with hypernatremia. On the basis of the clinical and biochemical findings the diagnosis of diabetes insipidus was made. Further evaluation revealed no anterior pituitary dysfunction. MRI of the sella showed pituitary stalk thickening (Fig3) and the diagnosis of possible hypophysitis was made. Due to the severity and the persistence of her symptoms she was also treated with 40 mgrs of prednozolone tapered gradually. Her symptoms gradually improved and her diabetes insipidus completely disappeared at 3 months. MRI of the pituitary at 3 months showed a normal pituitary stalk (Fig4).

Conclusion: Hypophysitis can have a variable clinical and imaging presentation. As in our cases, diabetes insipidus, although rare, can be one of the clinical presentations. Corticosteroid treatment can improve the clinical course and the imaging findings.

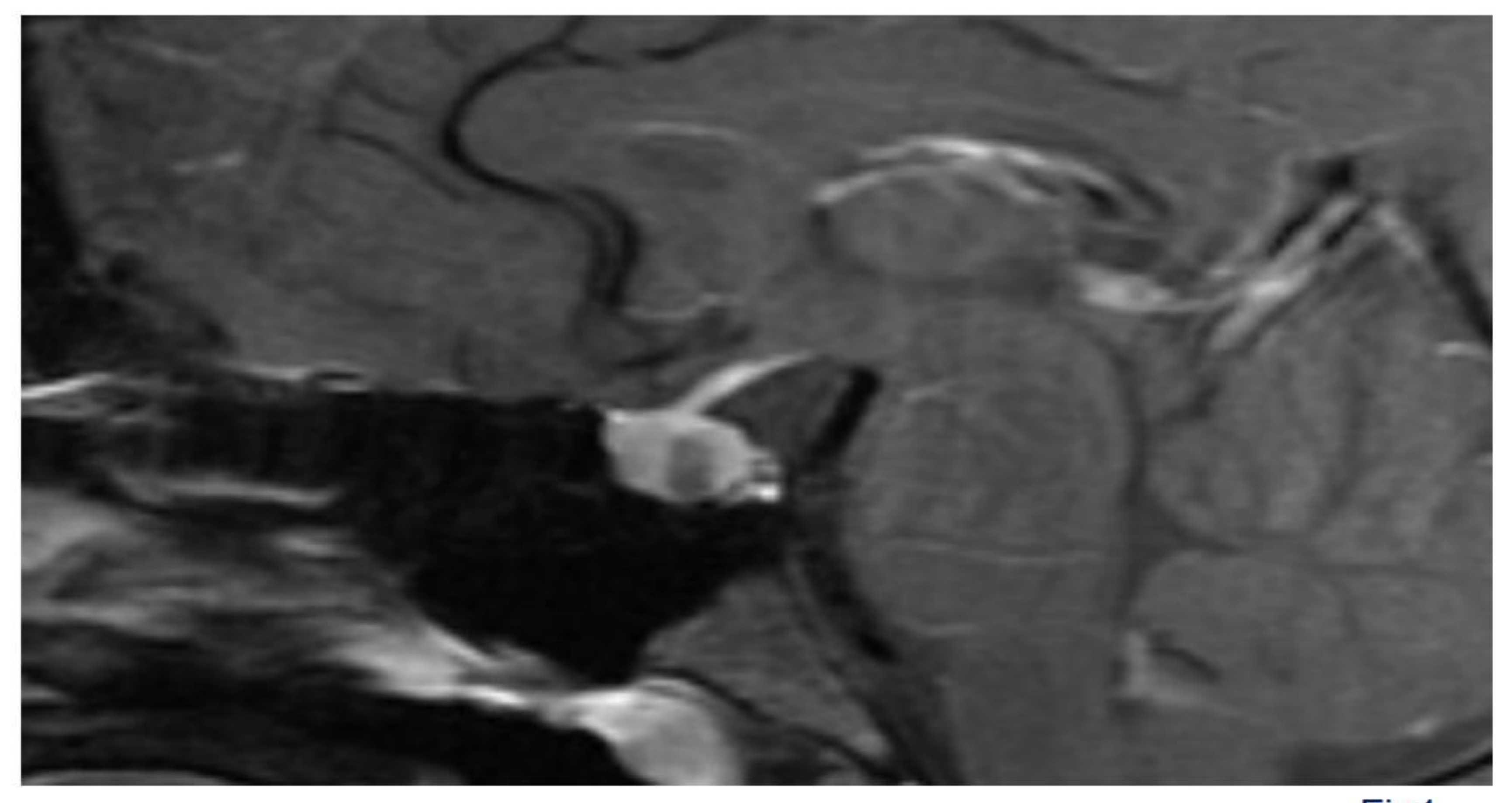

Fig1
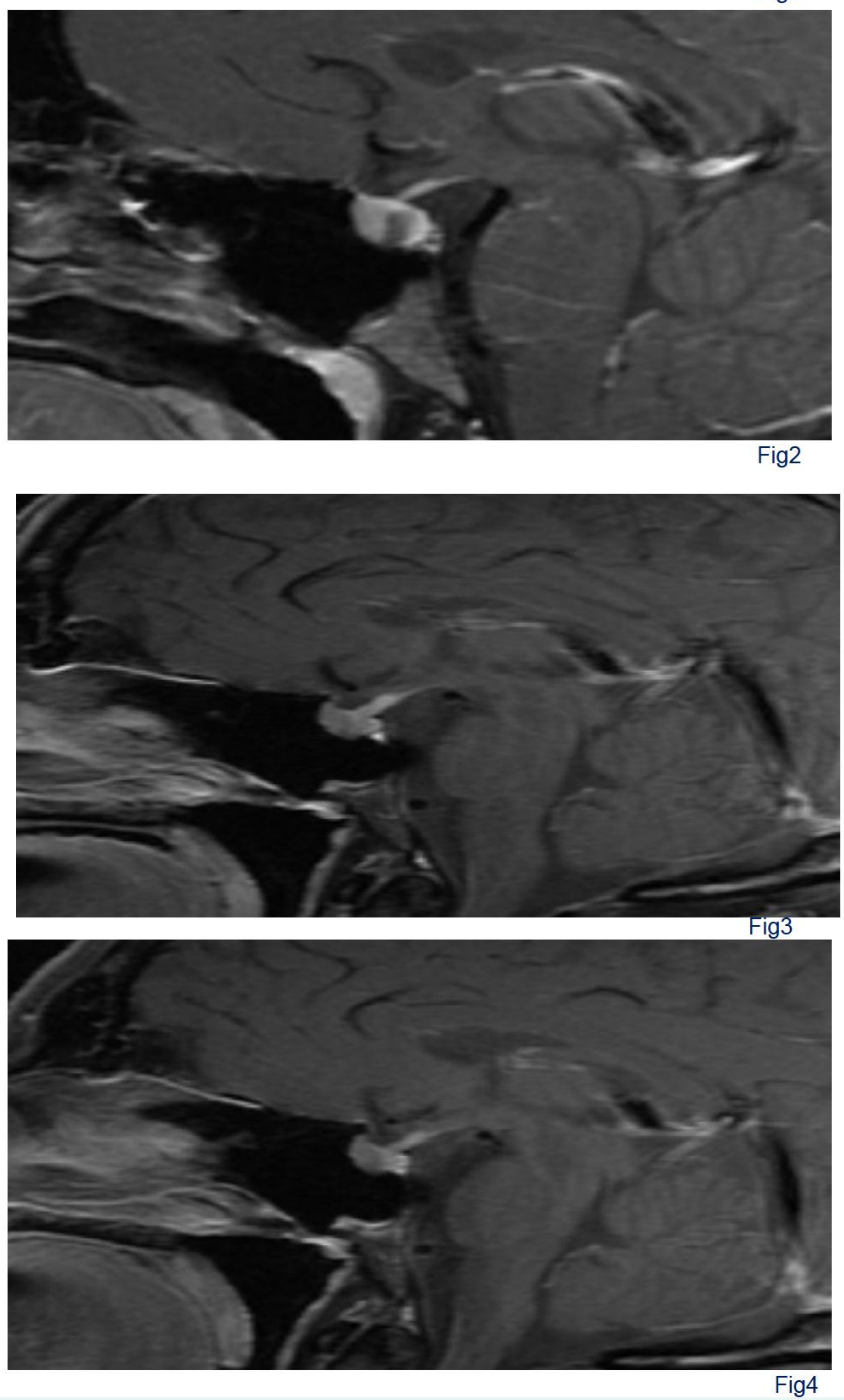

Referrences

Leporatiet al, JCEM, July 2011, 96(7):1971-1980

Caturegliet al, Endocrine Reviews, August 2005, 26(5):599-614 AutoimmmuneDiseases Coordinating Committee 2003 NIH

Shimatsuet al,EndocrineJournal 2009, 56 (9), 1033-1041 